\title{
A Self-tuning People Identification System from Split Face Components
}

\author{
Maria De Marsico ${ }^{2}$, Michele Nappi ${ }^{1}$, and Daniel Riccio ${ }^{1}$ \\ ${ }^{1}$ Universitá Degli Studi di Salerno, \\ via Ponte Don Melillo, 84084, Fisciano, Salerno, Italy \\ \{mnappi,driccio\}@unisa.it \\ ${ }^{2}$ Universitá Degli Studi di Roma - La Sapienza, \\ via Salaria 113, 00198, Roma, Italy \\ demarsico@di.uniroma1.it
}

\begin{abstract}
Multimodal systems can solve a number of problems found in unimodal approaches. We experimented going further along this line, by dividing the face into distinct regions (components) and processing each of them within a single subsystem. Such subsystems are then embedded in a more complex multicomponent architecture. In this way, typical tools of multimodal systems, such as reliability margins or fusion schemes, can be usefully extended to the single face biometry. An additional innovation element in this work is the definition of a global system auto-verification and auto-tuning policy able to produce a significant accuracy enhancement. The paper explores three integration architectures with different subsystem interconnection degree, demonstrating that a tight component interaction increases system accuracy and allows identifying unstable subsystems.
\end{abstract}

\section{Introduction}

The idea of considering the face as the union of distinct regions is not new in literature, and yet few significant examples exist at present. Poggio and Heisele deal with component-based face recognition [1], focusing on the pose problem. Face is partitioned into regions with limited overlap, each classified using linear operators, e.g. Principal Component Analysis (PCA) or Support Vector Machine (SVM). The superiority over global recognition systems suggests this as a solution for more face recognition problems, e.g. expression, lighting, or occlusions. However, such multicomponent approach inherits issues like normalization, fusion, and reliability of single responses. Most researches stop once regions and fusion modalities have been fixed, merely certifying the superiority of component-based approaches. An exception is [2] by Harandi et al., exploring the hierarchical combination of a global system with a component-based one, which activates when the former cannot return a response with a sufficient score. However, the component-based step is not even started if the first system chooses the wrong class, yet with high confidence. No work in present literature systematically investigates the different possibilities to combine single scores produced

T. Wada, F. Huang, and S. Lin (Eds.): PSIVT 2009, LNCS 5414, pp. $1-12,2009$.

(C) Springer-Verlag Berlin Heidelberg 2009 
by facial components, in terms of fusion levels, integration schemes, and reliability margins. The main contribution of this paper is therefore the definition of a framework integrating the main concepts of both component-based facial recognition and of multimodal authentication. The proposed system partitions the face image into its constituent regions. Afterwards, each of them is considered as a separate subsystem, which can be integrated with all the others in a multimodal schema. Issues introduced by such new perspective make up further investigation topics in this work. We chose AR-Faces database as a test-bed for our study, as it contains a sufficiently varied set of distortions and a number of subjects suited to the goal. As for the feature extraction process, we chose a fractal-based approach, which is robust to local distortions [3].

\section{Feature Extraction}

The algorithm exploited to extract features from face components is based on the Partitioned Iterated Function Systems (PIFS). In such technique, the original image $I$ is divided into a set $R=r_{1}, r_{2}, \ldots, r_{|R|}$ of disjoint square regions, called ranges, which are a covering of $I$. A set of larger regions $D=d_{1}, d_{2}, \ldots, d_{|D|}$ called domains, is extracted from $I$. The side length of a domain is twice that of a range. Domains can overlap, and are much more numerous than ranges. The image $I$ is encoded range by range; each range $r$ is approximated by a domain $d$ according to an affine transformation, whose parameters are computed by solving a least square problem. The approximating domain $d$ is chosen so as to minimize the quadratic error with respect to the Euclidean norm. PIFS have been adapted to recognize face [3, by encoding only a selected set of ranges. The original image is divided into interest regions, namely eyes, nose and mouth. Location of regions is performed using an object detector based on Haar features [4, and implemented in the OpenCV library [5] and exploiting data provided in [6], in particular Haar Cascades. A fixed number of entry points are identified on each region. Entry points, for example eye or mouth corners, are in the same positions for every subject. For each entry point in each region, the adopted algorithm extracts the range whose upper left corner corresponds to it, and approximates such range by a domain. In order to make the method robust to image shifts, ranges close to the the entry point are also considered and approximated. In a revised version of the technique, entry points are located on a fixed grid and this change provides better results. The best approximating domains are gradually clustered according to an on-line algorithm. The result obtained at the end of the feature extraction process is a list of centroids, each representing the characterizing element of a cluster of approximating domains. Each centroid $C$ stores three main pieces of information: the $C_{x}$ and $C_{y}$ coordinates and the $C_{s t d}$ variance, computed as the mean value of the corresponding feature of all domains in the cluster. As the algorithm progresses, domains are clustered according to their distance from the present centroids. A list of centroids is obtained for 
each interest region. For a discussion about algorithm parameters (number of range/domain, clustering options, number of centroids) see [3]). From this point on, we present three possible solutions for the recognition problem.

\section{The Integration Scheme}

Going further along the approach by Heisele and Poggio [1, we propose three architectures with increasing complexity, from a plain component-based method to a component-based self-tuning method). This last architecture, which is significantly different from the state of art in literature, will be presented in the next section. The algorithm implemented by the recognition systems to classify the single components is always the same presented above. Therefore, the reported different performances can be unequivocally ascribed to the peculiar features of the different architectures. This consideration adds value to the experimental results in terms of consistency and readability.

\subsection{The Plain Component-Based Protocol}

In the Plain Component-Based Protocol (PCBP), the lists of centroids obtained through PIFS for the four face interest regions are chained in a single feature vector $V$, characterizing the whole face. We have a single recognition module. Such approach is comparable to component-based methods in literature [1. A query image undergoes the same process, and the recognition module compares its feature vector with stored ones. The comparison between vectors $V_{1}$ and $V_{2}$ exploits an ad hoc distance function. For each centroid $C(x, y) \in V_{1}, V_{2}$ is searched for the centroid $\bar{C}(x, y)$ that is closest to $C(x, y)$ with respect to Euclidean distance, and the difference $\left|C_{s t d}-\bar{C}_{s t d}\right|$ is computed to obtain a difference vector $V_{\text {diff }}$. The average value $V_{\text {diff }}^{-}=E\left[V_{\text {diff }}\right]$ of the vector components is computed, and all the values in $V_{\text {diff }}$ higher than $V_{\text {diff }}^{-}$are discarded. In this way, the algorithm is made more robust with respect to occlusions. A detailed discussion about the computed vector distance can be found in $[3]$.

\subsection{The Parallel Protocol}

The first evolution takes to the Parallel Protocol (PP). PIFS are applied to each face region, as in Section 3.1, except that the four feature vectors are not chained. Each one is stored in a distinct database (left eyes, right eyes, etc.). Each component feeds an independent subsystem. In the same way, a query image is divided into the four relevant regions. The four corresponding component subsystems work in parallel and independently, each producing a list of subjects from its specific database; list elements include the ID of a database subject and a numeric score expressing its similarity with the input. Each list is ordered by such similarity, then the four lists are processed by a fusion module to obtain the global response. Such procedure allows further differences from the base schema in Section 3.1. because two elements, which are peculiar of multimodal systems, can be imported here in a component-based system: 
- Reliability Margins: Subsystems might not be equally reliable, due to the possibly different quality of face regions from time to time. An unreliable response is a valid reason to perform a further check. The definition of a measure for response reliability is then a crucial step for fusion. Some solutions use margins, measuring the "risk" associated to a single subsystem response after observing its scores. Poh and Bengio [7] introduce a confidence margin based on False Acceptance Rate (FAR) and False Rejection Rate (FRR). Many responses are marked as reliable, as the margin relies on an estimate of the actual distribution of genuine/impostor subjects' scores. This might be inappropriate when very high security is required. Moreover, frequentist approaches assume that the scores of the testing and development sets always originate from similar distributions. We adopt the new System Response Reliability (SRR) margin [8], based on a system/gallery dependent metric, and measuring the ability of separating genuine subjects from impostors on a single probe basis. Therefore, in our approach, each subsystem $T_{k}$ produces a reliability measure $\operatorname{srr}_{k, i}$ for each response $s_{k, i}$. Moreover, each subsystem $T_{k}$ is characterized by a threshold $t h_{k}$, such that a response $s_{k, i}$ is considered as reliable only if $s r r_{k, i} \geq t h_{k}$.

- Fusion Policies: Different policies exist for fusion. Veeramachaneni et al 9 investigate decision level fusion in a binary hypotheses-testing problem, and in particular the selection of the best subset from different fusion rules, to optimize FAR or FRR. Apart from the limitations of the approach, the interesting observations are that, for 2 classifiers fusion, AND and OR rules constitute a very important set (out of 16 possible ones), and that, as the number of classifiers increases, the optimal fusion rules are constructed from these two, giving better results than Averaged Sum Rule. This suggests that, despite the variety of fusion policies, we can assume that AND and OR generally represent a suitable choice. In our case, we have a system $S$ composed by 4 subsystems $T_{k},(k=1,2,3,4)$, processing right eye, left eye, nose and mouth respectively, each exploiting a gallery $G_{k},(k=1,2,3,4)$ of components for the same number of identities. Notice that each identity in the set $H$ of enrolled subjects cane have more than one image in each single gallery. Our fusion rules exploit the above mentioned System Response Reliability (SRR). Each subsystem is able to compute a value $\operatorname{srr}_{k, i}$ estimating the reliability associated to its $i$-th response. Such values are normalized in the range $[0,1]$ for comparability. As discussed above, for each subsystem $T_{k}$ a threshold $t h_{k}$ is estimated, and possibly updated [8, above which its reliability is satisfactory enough. As for $O R$, the combined response is valid if at least one subsystem response reliability is above the corresponding threshold; the system returns the identity returned by the subsystem with the higher reliability above the corresponding threshold. In $A N D$ policy, the combined response is valid only if all reliabilities are above the corresponding thresholds; the system returns the identity with the minimum weighted sum of distances from the probe, where weights are the reliability indexes. 
We still have to determine the single thresholds $t h_{k}$. They can be fixed in advance, and remain fixed in time, or they can be computed and updated according to obtained responses. A compromise between the number of reliable responses and the system error rate must be obtained. Too high thresholds make the system too restrictive, with a low error rate but also a low number of acceptances, while too low ones risk canceling the advantages of the reliability measure. Assume that $T_{k}$ has executed $M$ times, producing the set $\left\{s_{k, 1}, \ldots, s_{k, M}\right\}$ of responses. The corresponding reliability measures are combined in a set $R H=\left\{s r r_{k, 1}, \ldots, \operatorname{srr}_{k, M}\right\}$ containing the history of system behaviour (reliability history). The value to assign to $t h_{k}$ is strictly correlated to $R H$ features, in particular to the average and to the variance 8 of its elements. A high $E[R H]$ means that system responses are generally reliable and that the corresponding threshold can be proportionally high. On the other hand, the variance $\sigma[R H]$ measures the stability of $T_{k}$. The best situation is then when $R H$ elements have a high average and a low variance, so that it is possible to fix a high value for $t h_{k}$. We can summarize the above observations in the formula:

$$
t h_{k}=\frac{E[R H]^{2}-\sigma[R H]}{E[R H]},
$$

The system, in particular $t h_{k}$ thresholds, has been adjusted according to a set of faces with similar features to those of the set used for identification tests; the two sets are different and disjoint.

\section{A Self-tuning Architecture: The Supervised Protocol}

A further re-examination of the classical component-based schema takes to system self-tuning, with a much more flexible and robust architecture. The main limit of architectures in Section 3 is that they do not seize the main advantage of considering the facial identification system as a multicomponent architecture. Each component works independently and final results give no feedback for the overall system. Formula 1 is good for computing and updating $t_{k}$ thresholds for single reliability indexes, according to the corresponding subsystem behaviour; however, it only accounts for that subsystem. On the contrary, assume the existence of a supervisor module (Supervised Protocol - SP) exploiting single subsystem responses and their reliability to compute the final global response, and using the latter to evaluate the overall system state and update its parameters (Fig. 1). Such module would implement an algorithm to update single thresholds also according to the behavior of the other subsystems, so converging to an optimal configuration independently from the starting $\left\{t h_{1}, t h_{2}, t h_{3}, t h_{4}\right\}$ configuration. The algorithm distinguishes two cases:

- No identity is retrieved with more votes than the others, i.e. more identities $I_{j}, j \in\{1,2, \ldots,|H|\}$ share the same number of votes. Notice that this case also applies when retrieved identities are all different, with 1 vote each. If at least one $T_{k}$ in any such group has $S R R_{k}>t h_{k}$, the response is marked as 


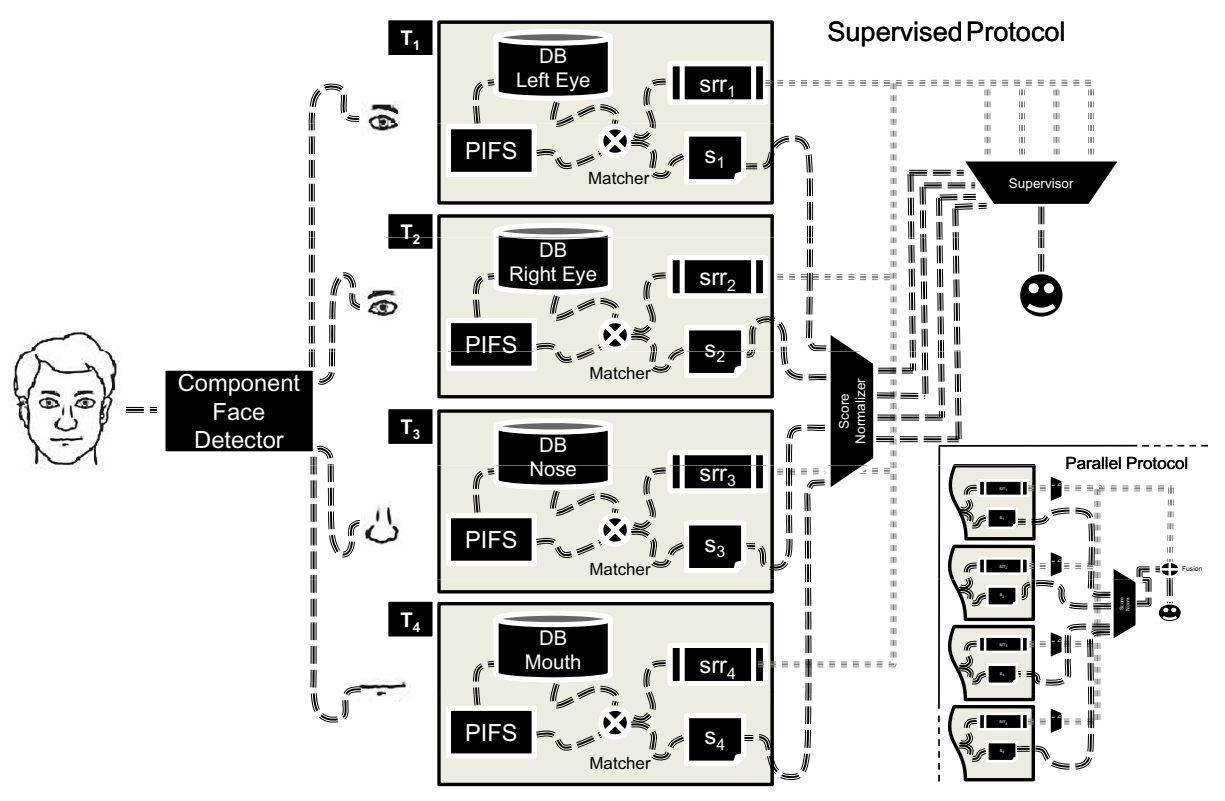

Fig. 1. The architecture of the supervised protocol

reliable, and the system returns the identity retrieved by the subsystem with the higher $S R R_{k}>t h_{k}$, otherwise the response is marked as unreliable.

- One identity $I_{j}$ gets more votes than the others. $I_{j}$ is returned and the response is marked as reliable.

In both cases, if the response is reliable, each subsystem $T_{k}$ voting for the returned identity is rewarded by lowering its threshold $t h_{k}$ by an updating step $u s$, unless its current $s r r_{k}$ is already above $t h_{k}$. Each other subsystem $T_{k}$ is penalized by increasing its threshold $t h_{k}$ by the updating step $u s$, unless its current $s r r_{k}$ is already below the respective $t h_{k}$. In this way the supervisor module lowers thresholds of subsystems voting in agreement, considering such behavior a confirmation of reliability, and increases thresholds of discordant ones, compensating possible distortions (local persistent distortions like lighting variations, dirt on lens). Pseudo-code of the resulting supervised face protocol in Fig. 2,

Such an architecture does not need an adjustment phase, since the system can start from a default configuration of its parameters and converge in any case towards an optimal one. The speed to reach such latter configuration is a significant system feature, so that it is important to define how to measure it. As we want to simulate the dynamic behavior of an online identification system, we assume that system time is beaten by performed recognition operations; we define a probe sequence $P=\left\{p_{1}, p_{2}, \ldots, p_{n}\right\}$ as a series of $n$ probes presented to the system, sharing the same acquisition characteristics (normal conditions, right light, glasses, scarf, dirty lens). A system equilibrium state (steady state) is given by the consecutive instants when threshold fluctuations are lower than a 


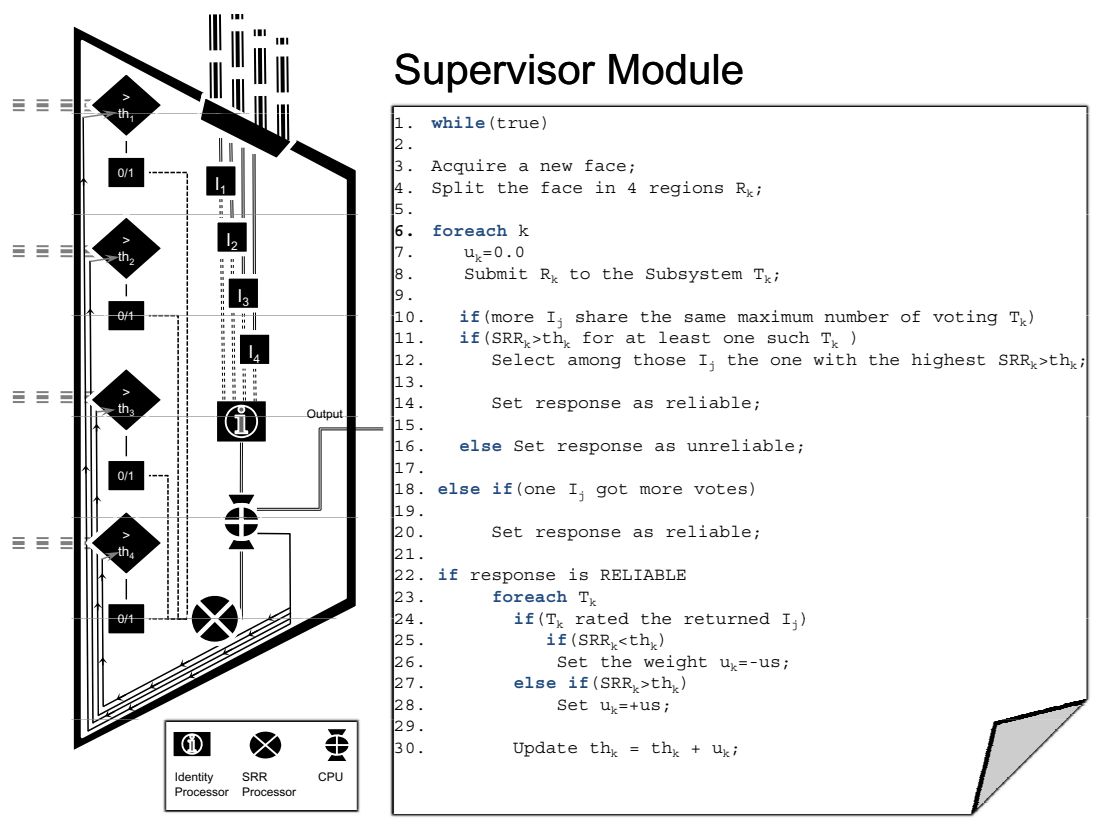

Fig. 2. The pseudo-code of the Supervisor Module

fixed $\mu$, while convergence speed of a subsystem $\lambda_{k}$ is defined as the ratio between the total variation of its threshold and the number of instants needed to obtain such transition. Total system convergence speed is defined as the minimum speed among all its subsystems, i.e. $\lambda=\min _{k} \lambda_{k}, k \in\{1,2,3,4\}$.

\section{$5 \quad$ Experimental Results}

Our tests exploited AR-Faces database [10. It consists of 126 persons (70 males and 56 females), each acquired in two different sessions with 13 image sets each. Sets differ in expression (1 neutral, 2 smile, 3 anger, 4 scream), illumination (5 left light, 6 right light, 7 all side light), presence/absence of occlusions ( 8 sun glasses, 11 scarf), or combinations (9 sun glasses and left light, 10 sun glasses and right light, 12 scarf and left light, 13 scarf and right light). Sets 14 to 26 of second session present the same conditions as 1 to 13 . Neutral images from the set 1 have been considered as the system gallery. Seven probe sets $(2,3,4,5,6,8$, 11) have been used for testing. Adopted measures for accuracy are Recognition Rate (RR), Equal Error Rate (EER) and Number of Reliable Responses (NRR).

\subsection{Comparisons among the Three Architectures}

In examining results, bear in mind that we are applying a decomposition to a biometry (the face) which is usually processed as a single overall recognition 
feature. We chose different test configurations with probe sequences varying in distortion typology. Notice that PCBP was used without SRR support (responses are always considered as reliable), and that PP was augmented with SRR using AND policy as in Section 3.2 .

In most cases, PP offers worse performances than PCBP, which is in general robust to occlusions and local distortions. Such result can be ascribed to the fact that single subsystems do not have any information about all the others. In SP, the supervisor module collects information from different subsystems and accordingly changes their parameters, so that they are influenced by what is happening within the global system. Such stronger interconnection among subsystems should increase system accuracy, as confirmed by experimental results.

To clarify the interpretation of the experiments, we notice that each of the selected AR-Faces sets can be considered as a sequence $P$ of test images, sequentially submitted to the system. The initial thresholds configuration is set at $\left\{t h_{1}=0.0, t h_{2}=0.0, t h_{3}=0.0, t h_{4}=0.0\right\}$, i.e. all responses are initially considered as reliable. The updating step us is fixed at 0.05. Table 1 shows that RR obtained with $\mathrm{SP}$ is comparable, or in some cases even better, than with PCBP, also obtaining a lower EER. Notice that the Number of Reliable Responses (NRR) obtained with PCBP is always equal to the total number of responses, as no SRR is used. We focus attention on set 4 . In this case the number of reliable responses is quite low, only 50 , but such as to guarantee a $\mathrm{RR}$ of 0.76 , definitely higher than that obtained with the global method. If we consider the sets of equilibrium thresholds (last four columns) reached by the system for sets 2, 3 and 4 , we can see that nose and mouth, as highly unstable regions, often disagreeing with the other subsystems, are penalized with much higher thresholds $\left(t h_{3}\right.$ and $\left.t h_{4}\right)$ for reliability indexes. The behavior on sets 5 and 6 confirms what stated above, as SP is able to reach higher RR values, yet with a lower EER and a quite high number of reliable responses. The sets of equilibrium thresholds reached by the system perfectly agree with the variations

Table 1. Performance comparison on different probes from AR-Faces among the three architectures: Plain Component-Based Protocol (PCBP), Parallel Protocol (PP) and Supervised Protocol (SP)

\begin{tabular}{|c|c|c|c|c|c|c|c|c|}
\hline \multirow{3}{*}{\multicolumn{2}{|c|}{ SubSET }} & \multicolumn{7}{|c|}{ EXPRESSION VARIATIONS } \\
\hline & & \multirow{2}{*}{ PCBP } & \multirow{2}{*}{ PP } & \multicolumn{5}{|c|}{$\begin{array}{ll}\text { SP } \\
\end{array}$} \\
\hline & & & & PERF. & $\mathrm{th}_{1}$ & $\mathrm{th}_{2}$ & $\mathrm{th}_{3}$ & $\mathrm{th}_{4}$ \\
\hline \multirow{3}{*}{$\begin{array}{c}\text { SET } \\
2\end{array}$} & RR & 0.92 & 0.89 & 0.94 & \multirow{3}{*}{0.15} & \multirow{3}{*}{0.30} & \multirow{3}{*}{0.40} & \multirow{3}{*}{0.70} \\
\hline & EER & 0.07 & 0.05 & 0.03 & & & & \\
\hline & NRR & 126 & 38 & 120 & & & & \\
\hline \multirow{3}{*}{$\begin{array}{c}\text { SET } \\
3\end{array}$} & RR & 0.95 & 0.98 & 0.94 & \multirow{3}{*}{0.43} & \multirow{3}{*}{0.42} & \multirow{3}{*}{0.95} & \multirow{3}{*}{0.58} \\
\hline & EER & 0.05 & 0.03 & 0.03 & & & & \\
\hline & NRR & 126 & 56 & 125 & & & & \\
\hline \multirow{3}{*}{$\begin{array}{c}\text { SET } \\
4\end{array}$} & $\mathrm{RR}$ & 0.48 & 0.36 & 0.76 & \multirow{3}{*}{0.1} & \multirow{3}{*}{0.40} & \multirow{3}{*}{0.73} & \multirow{3}{*}{0.72} \\
\hline & EER & 0.15 & 0.29 & 0.12 & & & & \\
\hline & NRR & 126 & 33 & 50 & & & & \\
\hline
\end{tabular}

\begin{tabular}{|c|c|c|c|c|c|c|c|c|}
\hline \multirow{3}{*}{\multicolumn{2}{|c|}{ SUBSET }} & \multicolumn{7}{|c|}{ ILLUMINATION VARIATIONS } \\
\hline & & \multirow{2}{*}{ PCBP } & \multirow{2}{*}{ PP } & \multicolumn{5}{|c|}{$\begin{array}{ll}\text { SP } \\
\end{array}$} \\
\hline & & & & PERF. & $\mathrm{th}_{1}$ & $\mathrm{th}_{2}$ & $\mathrm{th}_{3}$ & $\mathrm{th}_{4}$ \\
\hline \multirow{3}{*}{$\begin{array}{c}\text { SET } \\
5\end{array}$} & RR & 0.92 & 1.00 & 0.96 & \multirow{3}{*}{0.68} & \multirow{3}{*}{0.38} & \multirow{3}{*}{0.65} & \multirow{3}{*}{0.64} \\
\hline & EER & 0.03 & 0.02 & 0.02 & & & & \\
\hline & NRR & 126 & 30 & 112 & & & & \\
\hline \multirow{3}{*}{$\begin{array}{c}\text { SET } \\
6\end{array}$} & $\mathrm{RR}$ & 0.94 & 0.97 & 0.96 & \multirow{3}{*}{0.35} & \multirow{3}{*}{0.75} & \multirow{3}{*}{0.62} & \multirow{3}{*}{0.75} \\
\hline & EER & 0.05 & 0.07 & 0.03 & & & & \\
\hline & NRR & 126 & 37 & 107 & & & & \\
\hline \multirow{3}{*}{\multicolumn{2}{|c|}{ SubSET }} & \multicolumn{7}{|c|}{ OCCLUSIONS } \\
\hline & & \multirow{2}{*}{ PCBP } & \multirow{2}{*}{ PP } & \multicolumn{5}{|c|}{$\begin{array}{ll}\mathrm{SP} \\
\end{array}$} \\
\hline & & & & PERF. & $\mathrm{th}_{1}$ & $\mathrm{th}_{2}$ & $\mathrm{th}_{3}$ & $\mathrm{th}_{4}$ \\
\hline \multirow{3}{*}{$\begin{array}{c}\text { SET } \\
8\end{array}$} & RR & 0.71 & 0.25 & 0.98 & \multirow{3}{*}{0.65} & \multirow{3}{*}{0.60} & \multirow{3}{*}{0.60} & \multirow{3}{*}{0.55} \\
\hline & EER & 0.09 & 0.23 & 0.04 & & & & \\
\hline & NRR & 126 & 20 & 50 & & & & \\
\hline \multirow{3}{*}{$\begin{array}{c}\text { SET } \\
11\end{array}$} & RR & 0.85 & 0.61 & 0.92 & \multirow{3}{*}{0.45} & \multirow{3}{*}{0.40} & \multirow{3}{*}{0.84} & \multirow{3}{*}{0.90} \\
\hline & EER & 0.09 & 0.19 & 0.02 & & & & \\
\hline & NRR & 126 & 23 & 115 & & & & \\
\hline
\end{tabular}


introduced by the different sets of face images. Table 1 shows that the number of reliable responses for SP drops to 50 for sun glasses (set 8) and to 115 for scarf (set 11). This agrees with our expectations, as the distortions introduced involve a larger face area. However, out of a lower number of reliable responses, the system is able in both cases to guarantee a significantly higher accuracy than PCBP (RR of 0.98 versus 0.71 and of 0.92 versus 0.85 ) and lower EER. Even in this case the reached equilibrium thresholds are consistent with variations. As expected, PCBP performances are quite constantly worse than those obtained with SP. We can observe that, even when the accuracy of SP drops slightly below that of PP (sets 5 and 6), this is counterbalanced by a much higher number of reliable responses. As an overall consideration, the increase of accuracy possibly obtained by PP, due to the use of AND fusion policy, is almost always not worth the higher loss of results.

\subsection{Further Experiments on the Supervised Protocol}

The first experiment in this section aims at demonstrating that the behavior of the algorithm for threshold updating is stable. In other words, for different probe sequences $P_{j}$, all composed by images with the same kind of distortion (smile, right light, sun glasses, dirty lens), the values for the thresholds converge towards the same set, with a small margin of variation. For this experiment, set 1 is used as gallery, while probe sequences are extracted from set 2, 6 and 11 . Each probe sequence is built by randomly extracting 1000 times one of the 126 images from the probe set; Fig. 3 shows the results for set 2 . We can observe that thresholds $t h_{1}$ and $t h_{2}$ (right and left eye) tend to assume lower values than $t h_{3} \mathrm{e}$ $t h_{4}$ (nose and mouth). The latter values show an initial variation, and then stay constant for all the remaining part of the probe sequence. This can be explained by observing that, since images in set 2 belong to smiling subjects, nose and
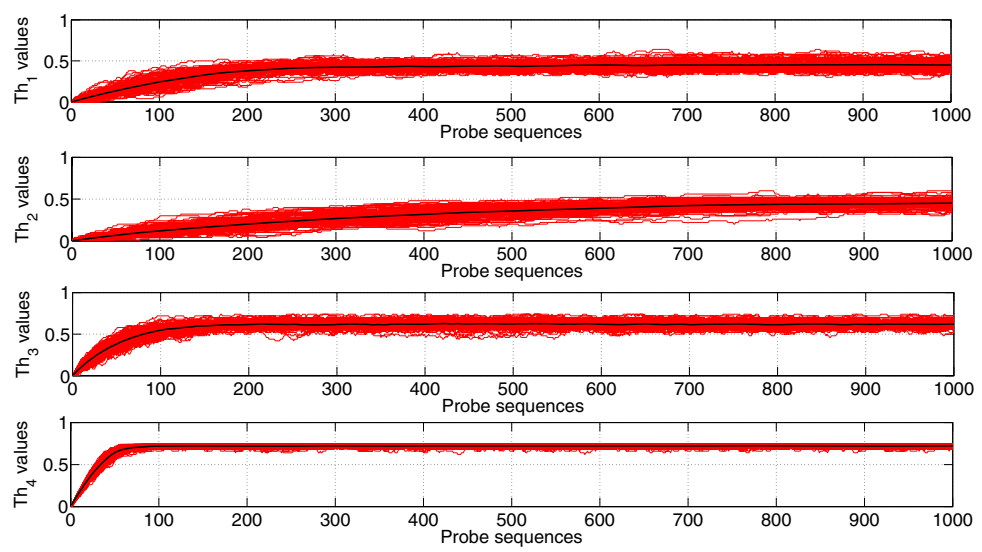

Fig. 3. Variation of thresholds of subsystems $T_{1}, T_{2}, T_{3}$ and $T_{4}$ for 100 probe sequences of 1000 images of 126 smiling subjects (set 2); the black curve represents the mean trend 

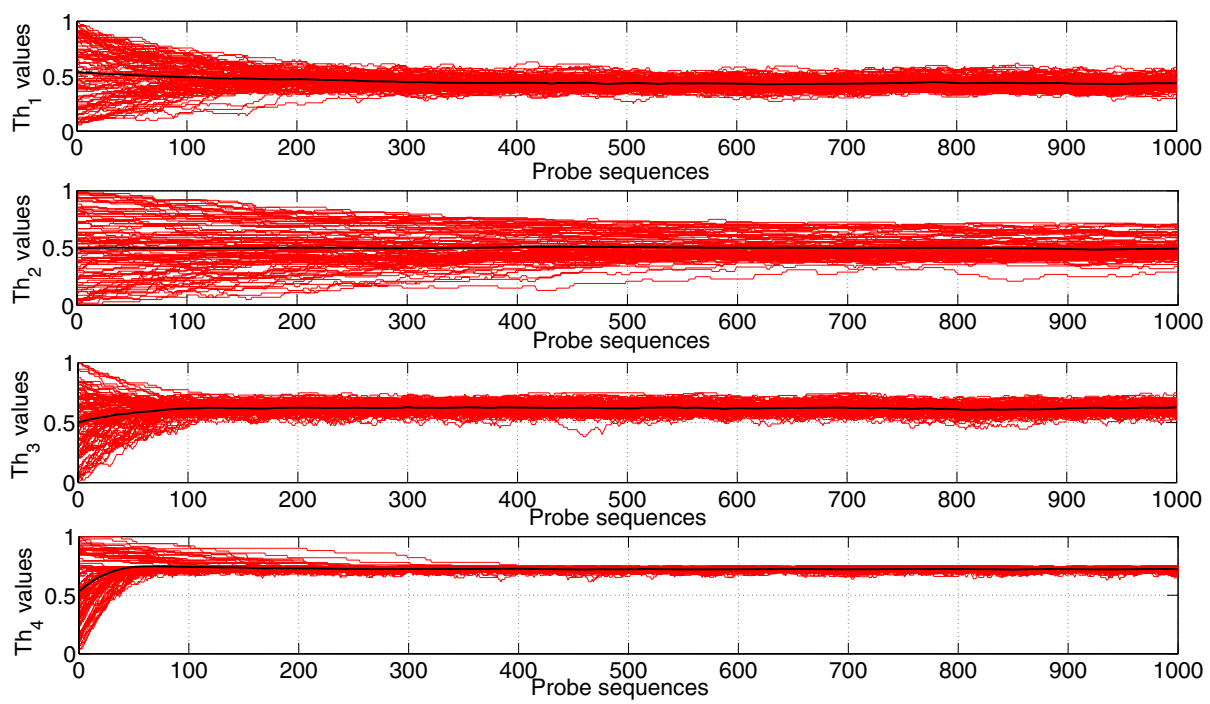

Fig. 4. Variation of thresholds of subsystems $T_{1}, T_{2}, T_{3}$ and $T_{4}$ for 100 probe sequences of 1000 images of 126 smiling subjects (set 2) according to the initial thresholds configuration $\left(\left\{t h_{1}, t h_{2}, t h_{3}, t h_{4}\right\}\right)$; the black curve represents the mean trend

mouth show an higher variability than eyes, making the corresponding systems $T_{3}$ e $T_{4}$ less reliable, and therefore demanding higher values for the respective thresholds. The darker line (in black) in Fig. 3 is the mean value of the 100 computed curves and represents the mean trend for thresholds variation. For each threshold, mean $(\{0.40,0.32,0.59,0.70\})$ and variance $(\{0.10,0.12,0.09$, $0.09\}$ ) of the values in the 100 iterations are also computed; the same for the Recognition Rate (mean: 0.94, variance: 0.0072) and the Number of Reliable Responses (mean: 955, variance: 6.2261).

The second experiment aims at highlighting how the threshold configuration towards which the system converges is independent from the starting configuration. Even in this case, we considered 100 probe sequences of 1000 images randomly extracted among the 126 of set 2 . For each system run, the initial values for thresholds are randomly chosen (all values are equally probable) in the interval $[0,1]$. As can be observed from Fig. 4, which reports the results of experiments on set 2 for each threshold, the curves generated by the different probe sequences tend to always concentrate in a relatively small final interval. This confirms the convergence of the updating procedure. As for the preceding experiment, mean $(\{0.45,0.50,0.62,0.72\})$ and variance $(\{0.03,0.00,0.02,0.02\})$ of values in the 100 iterations are computed; the same for the Recognition Rate (mean: 0.94, variance: 0.0072) and the Number of Reliable Responses (mean: 951, variance: 6.7054 ). We can observe that variance of the single thresholds is lower than in the preceding experiment; this is imputable to the absence of the strong initial variation in the first stretch of the curves in Fig. 3. Fig. 5 shows the thresholds variation over a probe sequence composed of 5 subsequences of 


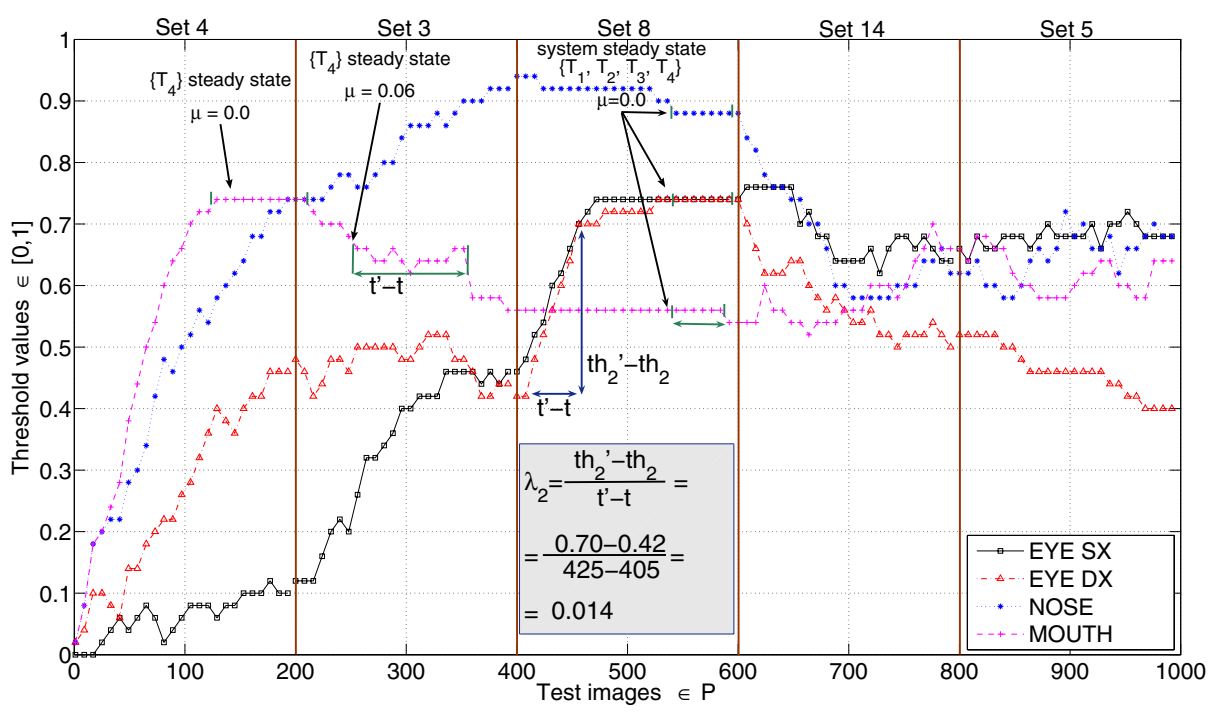

Fig. 5. Example of thresholds variation over a probe sequence of 1000 images from set 4 (1 - 200), 3 (201 - 400), 8 (401 - 600), 14 (601 - 800) and 5 (801 - 1000)

200 images each. Each subsequence is composed of randomly extracted images from sets 4, 3, 8, 14 and 5. Notice that for the first sequence the thresholds $t h_{3}$ e $t h_{4}$ reach higher values than the others, consistently with the fact that in images from set 4 mouth presents the higher variability. The configuration undergoes a reversal of trend in the third sequence, corresponding to set 8 , where the presence of sun glasses lowers reliability of subsystems $T_{1}$ and $T_{2}$. Afterwards it returns to a rather balanced trend on set 14, where no particular expression or lighting variations are present. Lighting variations, and more precisely left light in set 5 , lower performances of $T_{1}, T_{3}$ and $T_{4}$ subsystems, while the only one to remain more reliable is $T_{2}$. Fig. 5 shows some examples of the computation of the subsystems convergence speed (for $T_{2}$ ) and of the identification of equilibrium states with different values of $\mu\left(T_{4}\right)$; it is to also to notice a case when all the subsystems are in equilibrium, so that we can speak about system equilibrium.

\section{Conclusions}

We faced the problem of face recognition by a component-based system. Such system integrates peculiar multimodal tools as reliability margins and fusion policies. We discuss three different integration schemes: plain component based protocol, parallel protocol and supervised protocol. They mainly differ for the level of cohesion among subsystems. Experimental results show how a high interconnection degree improves the global system performances in terms of accuracy. Along this line, we can set suitable extensions to properly multimodal systems [1]. 


\section{References}

1. Heisele, B., Ho, P., Wu, J., Poggio, T.: Face recognition: component-based versus global approaches. Computer Vision and Image Understanding 91, 6-21 (2003)

2. Harandi, M.T., Ahmadabadi, M.N., Araabi, B.N.: A hierarchical face identification system based on facial components. In: Proceedings of the IEEE/ACS International Conference on Computer Systems and Applications, pp. 669-675 (2007)

3. Abate, A.F., Nappi, M., Riccio, D., Tucci, M.: Occluded face recognition by means of the IFS. In: Kamel, M.S., Campilho, A.C. (eds.) ICIAR 2005. LNCS, vol. 3656, pp. 1073-1080. Springer, Heidelberg (2005)

4. Viola, P., Jones, M.: Rapid object detection using a boosted cascade of simple features. In: Proceedings of the IEEE Computer Society Conference on Computer Vision and Pattern Recognition (CVPR), pp. 511-518 (2001)

5. Open Source. Open source computer vision library (2006), http://sourceforge.net/projects/opencvlibrary/

6. Bhatti, Z.E.: Face and eyes detection using opencv (2008), http: //www . codeproject. com/KB/library/eyes . aspx? $\mathrm{fid}=990485 \& d f=90 \&$ $\mathrm{mpp}=25 \&$ noise=3\&sort=Position\&view=Quick\&select $=2514967 \& \mathrm{fr}=26$

7. Poh, N., Bengio, S.: Improving fusion with margin-derived confidence in biometric authentication tasks. In: Kanade, T., Jain, A., Ratha, N.K. (eds.) AVBPA 2005. LNCS, vol. 3546, pp. 474-483. Springer, Heidelberg (2005)

8. Abate, A.F., Nappi, M., Riccio, D., De Marsico, M.: Data normalization and fusion in multibiometric systems. In: Proceedings of the 12th International Conference on Distributed Multimedia Systems (DMS 2007), pp. 87-92 (2007)

9. Veeramachaneni, K., Osadciw, L., Varshney, P.K.: An adaptive multimodal biometric management algorithm. IEEE Transaction on Systems, Man, and CyberneticsPart C: Applications and Reviews 35, 344-356 (2005)

10. Martinez, A.M.: Recognizing imprecisely localized, partially occluded, and expression variant faces from a single sample per class. IEEE Transaction on Pattern Analisys and Machine Intelligence 24, 748-763 (2002)

11. Abate, A.F., Nappi, M., Riccio, D., De Marsico, M.: Face, ear and fingerprint: Designing multibiometric architectures. In: Proceedings of the 14th International Conference on Image Analysis and Processing (ICIAP 2007), pp. 437-442 (2007) 